Pacific Journal of Mathematics

ON HAUSDORFF COMPACTIFICATIONS 


\title{
ON HAUSDORFF COMPACTIFICATIONS
}

\author{
MARLON C. RAYBURN
}

Given a pair of spaces $X$ and $Y$, a necessary and sufficient condition is found for $Y$ to be homeomorphic to $\operatorname{cl}_{\alpha X}(\alpha X-X)$ for some compactification $\alpha X$ of $X$. From this follows a necessary and sufficient condition for $Y$ to be homeomorphic to $\alpha X-X$ for some $\alpha X$. As an application, a sufficient condition is found to insure the isomorphism of the upper semi-lattices of compactifications $K(X)$ and $K(Y)$ for arbitrary $X$ and $Y$, and in consequence it appears that for every space $X$, there is a pseudocompact space $Y$ with $K(X)$ isomorphic to $K(Y)$. A necessary condition for $K(X)$ to be isomorphic to $K(Y)$ is observed for arbitrary $X$ and $Y$, and this leads to the consideration of spaces compactly generated at infinity. Examples are constructed.

All spaces considered are completely regular and Hausdorff. We consider the family of Hausdorff compactifications of $X$, each obtained by a quotient map on $\beta X$ fixing $X$ pointwise. It is known [3: 10.13] that this map, hereafter called the "Čech map" of the compactification, must be unique. Identify any two such compactifications if there is a homeomorphism between them which fixes $X$ pointwise and let $K(X)$ be the family of equivalence classes partially ordered in the standard way: $\alpha_{1} X \leqq \alpha_{2} X$ if there is a continuous map from $\alpha_{2} X$ onto $\alpha_{1} X$ which fixes $X$ pointwise. From [2], $K(X)$ is an upper semi-lattice which is a complete lattice if and only if $X$ is locally compact. In [5] K. D. Magill, Jr. obtained the result which shall be referred to as Magill's theorem: For any two locally compact spaces $X$ and $Y, K(X)$ is lattice-isomorphic to $K(Y)$ if and only if $\beta X-X$ is homeomorphic to $\beta Y-Y$.

In this paper, generalizations are obtained to each direction of Magill's theorem by dropping the requirement that $X$ and $Y$ be locally compact.

\section{Compactifications.}

Lemma 1.0. Let $X$ be a compact Hausdorff space, $Y$ be a compact $T_{1}$ space and $f: X \rightarrow Y$ be continuous and onto. The following are equivalent:

(a) $Y$ is Hausdorff

(b) $f$ is closed

(c) For every $p \in Y$ and for all open sets $U \subseteq X$ such that $f^{\leftarrow}(p) \leqq U$, there is an open set $V \leqq Y$ with $p \in V$ and $f^{\leftarrow}[V] \subseteq U$. 
For any space $X$, let $R(X)$ be the set of all points at which $X$ is not locally compact. It follows that for any compactification $\alpha X$ of $X, R(X)=X \cap \mathrm{cl}_{\alpha X}(\alpha X-X)$.

THEOREM 1.1. Given any two spaces $X$ and $Y$, there is a compactification $\alpha X$ of $X$ such that $Y$ is homeomorphic to $\operatorname{cl}_{\alpha X}(\alpha X-X)$ if and only if there is a continuous map $h$ from $\operatorname{cl}_{\beta X}(\beta X-X)$ onto $Y$ such that $h$ is one-to-one on $R(X)$.

Proof. From the existence of the Čech map, the "only if" is trivial. Conversely with no loss of generality assume $Y$ and $X-R(X)$ to be disjoint and define $\alpha X$ to be the set $Y \cup X-R(X)$. Let $f: \beta X \rightarrow \alpha Y$ be given by $f(x)=x$ for $x$ in $X-R(X)$ and $f(x)=h(x)$ for $x$ in $\operatorname{cl}_{\beta X}(\beta X-X)$. Place the quotient topology of $f$ on $\alpha X$, which is thus a compact $T_{1}$ space containing $[X-R(X)] \cup h[R(X)]$ densely. We need to show $\alpha X$ to be Hausdorff, and shall use part (c) of the Lemma to do this.

First suppose $p \in X-R(X)$ and $U$ is an open set in $\beta X$ such that $f^{\leftarrow}(p)=\{p\} \subseteq U$. Let $V=[X-R(X)] \cap U$. Then $V$ is a $\beta X$ open set and $f^{-} \circ f[V]=V$. So $V=f[V]$ is open in $\alpha X, p \in V$ and $f^{\leftarrow}[V] \subseteq U$.

Now let $p \in \alpha X-[X-R(X)]$. Then $p \in Y$ and $f^{\leftarrow}(p)=h^{\leftarrow}(p)$ in $\operatorname{cl}_{\beta X}(\beta X-X)$. Let $U$ be any $\beta X$-open neighborhood of $h^{-}(p)$. Then $U \cap \operatorname{cl}_{\beta X}(\beta X-X)$ is an open set in $\operatorname{cl}_{\beta X}(\beta X-X)$ and contains $h^{-}(p)$. Since $h$ is a closed map, there exists a $Y$-open set $A$ such that $h^{-}(p) \subseteq h^{-}[A] \subseteq U \cap \operatorname{cl}_{\beta X}(\beta X-X)$. But considering $A$ as a set in $\alpha X-[X-R(X)], f^{\leftarrow}[A]=h^{\leftarrow}[A]$ is open in $\operatorname{cl}_{\beta X}(\beta X-X)$. So there exists a $\beta X$-open set $B$ such that $B \cap \operatorname{cl}_{\beta X}(\beta X-X)=f^{\leftarrow}[A]$. Let $G=B \cap U$, this is an open set in $\beta X$. Then $G \cap[X-R(X)] \subseteq U \cap$ $[X-R(X)]$ and $G \cap \operatorname{cl}_{\beta X}(\beta X-X)=f^{-}[A]$. Whence if we set $V=$ $A \cup[G \cap X-R(X)]$, we have $p \in V$ and

$$
\begin{aligned}
f^{\leftarrow}[V] & =f^{\leftarrow}[A \cup(G \cap X-R(X))]=f^{-}[A] \cup f^{\leftarrow}[G \cap X-R(X)] \\
& =\left[G \cap \operatorname{cl}_{\beta X}(\beta X-X)\right] \cup[G \cap X-R(X)]=G .
\end{aligned}
$$

Thus $V$ is open in $\alpha X$ and $f^{-}[V]=G \subseteq U$.

We conclude that $\alpha X$ is a compact Hausdorff space containing a dense homeomorphic image of $X$, and $f: \beta X \rightarrow \alpha X$ is its Čech map.

Finally, let $\tau: Y \rightarrow \mathrm{cl}_{\alpha X}(\alpha X-X)$ be given by $\tau(y)=f\left[h^{-}(y)\right]$ for each $y \in Y$. Since $h^{-}(y) \subseteq \operatorname{cl}_{\beta X}(\beta X-X)$, for each point $q \in h^{-}(y)$ we have $f(q)=h(q)=y$. So $\tau$ is well defined, and indeed it is a bijection. Moreover since $f$ and $h$ are closed maps, any set $F$ of $Y$ is closed if and only if $h^{-}[F]$ is closed in $\operatorname{cl}_{\beta X}(\beta X-X)$, which is true if and only if $f\left(h^{-}[F]\right)$ is closed in $\operatorname{cl}_{\alpha X}(\alpha X-X)$. Thus $\tau$ is a homeomorphism from $Y$ onto $\mathrm{cl}_{\alpha X}(\alpha X-X)$. 
COROLlary 1.2. For any space $X$, the following are equivalent:

(a) $X$ is locally compact.

(b) For every space $Y: Y$ is homeomorphic to $\alpha X-X$ for some Hausdorff compactification $\alpha X$ of $X$ if and only if $Y$ is a continuous image of $\beta X-X$.

Proof. For (b) implies (a), note that a map onto a single point is trivially continuous. For the converse, take $R(X)=\varnothing$ in the Theorem. The fact that (a) implies (b) was first observed in [4].

THEOREM 1.3. Let $X$ and $Y$ be any two spaces. There is a compactification $\alpha X$ of $X$ such that $Y$ is homeomorphic to $\alpha X-X$ if and only if there is a compactification $\alpha Y$ of $Y$ and a continuous map $h$ from $\operatorname{cl}_{\beta X}(\beta X-X)$ onto $\alpha Y$ such that h carries $R(X)$ homeomorphically onto $\alpha Y-Y$.

Proof. (If). By Theorem 1.1, there is a compactification $\alpha X$ of $X$ such that $\operatorname{cl}_{\alpha X}(\alpha X-X)$ is homeomorphic to $\alpha Y$. Moreover if $f: \mathrm{cl}_{\beta X}(\beta X-X) \rightarrow \mathrm{cl}_{\alpha X}(\alpha X-X)$ is the restriction of the Čech map, we may choose the homeomorphism $\tau: \operatorname{cl}_{\alpha X}(\alpha X-X) \rightarrow \alpha Y$ by $\tau(X)=$ $h\left[f^{\leftarrow}(X)\right]$ as in the final paragraph of Theorem 1.1. Since $\tau[R(X)]=$ $\alpha Y-Y$, we see that $\tau$ carries $\alpha X-X$ homeomorphically onto $Y$.

(Only if) Suppose that $h: \alpha X-X \rightarrow Y$ is the given homeomorphism. Without loss of generality assume $Y$ and $R(X)$ disjoint, and let $\alpha Y$ be the set $Y \cup R(X)$. Define $k: \operatorname{cl}_{\alpha X}(\alpha X-X) \rightarrow \alpha Y$ by $k(p)=p$ if $p \in R(X)$ and $k(p)=h(p)$ if $p \in \alpha X-X$. Place the quotient topology with respect to $k$ on $\alpha Y$, making $\alpha Y$ into a compact $T_{1}$ space.

If $F$ is any closed subset of $\operatorname{cl}_{\alpha X}(\alpha X-X)$, then since $k$ is a bijection, $k^{\leftarrow} \circ k[F]=F$ and $k[F]$ is closed in the quotient topology on $\alpha Y$. Hence $k$ is a homeomorphism between $\mathrm{cl}_{\alpha X}(\alpha X-X)$ and $\alpha Y$. So $\alpha Y$ is Hausdorff and $Y$, being the image of a dense subset of $\mathrm{cl}_{\alpha X}(\alpha X-X)$ is dense in $\alpha Y$. Thus $\alpha Y$ is a Hausdorff compactification of $Y$.

Let $f$ be the restriction to $\operatorname{cl}_{\beta X}(\beta X-X)$ of the Čech map of $\alpha X$. Then $k \circ f$ is continuous from $\operatorname{cl}_{\beta X}(\beta X-X)$ onto $\alpha Y$. But $k \circ f$ takes $\beta X-X$ onto $Y$ and also takes $R(X)$ one-to-one onto $\alpha Y-Y$, so it is a homeomorphism from $R(X)$ onto $\alpha Y-Y$.

COROLLARY 1.4. Let $X$ and $Y$ be any two spaces and $h$ be a homeomorphism from $\operatorname{cl}_{\beta X}(\beta X-X)$ onto $\operatorname{cl}_{\beta Y}(\beta Y-Y)$ which carries $R(X)$ onto $R(Y)$. Let $\alpha X$ be any compactification of $X$ and let $f$ be the restriction of its $\breve{C}$ ech map to $\operatorname{cl}_{\beta X}(\beta X-X)$. Then there exists a unique (up to a homeomorphism preserving $Y$ pointwise) compactification $\alpha Y$ of $Y$, with $\breve{C}$ ech map $g$, such that $g\left(h\left(f^{\leftarrow}(x)\right)\right)$ is a homeomor- 
phism from $\mathrm{cl}_{\alpha X}(\alpha X-X)$ onto $\mathrm{cl}_{\alpha Y}(\alpha Y-Y)$ taking $R(X)$ onto $R(Y)$.

2. The upper semi-lattice of compactifications. For each compactification $\alpha X$ of $X$, with Čech map $f$, define

$$
\mathscr{F}(\alpha X)=\left\{f^{\leftarrow}(p): p \in \operatorname{cl}_{\alpha X}(\alpha X-X)\right\} \text {. }
$$

This is a partition of $\operatorname{cl}_{\beta X}(\beta X-X)$ into compact subsets and coincides with Magill's terminology on locally compact spaces [5]. In particular, we retain his

LEMmA 2.1. $\alpha_{1} X \leqq \alpha_{i} X$ if and only if $\mathscr{F}\left(\alpha_{2} X\right)$ refines $\mathscr{F}\left(\alpha_{1} X\right)$. Observe that in $K(X)$, the correspondence between compactifications and their decompositions is one-to-one.

Let $X$ and $Y$ be any spaces and $K(X)$ and $K(Y)$ be their upper semi-lattices of compactifications. We say $K(X)$ is isomorphic to $K(Y)$ if there is a bijection between them which preserves order in both directions. Clearly an isomorphism preserves meets and joins wherever they exist.

THEOREM 2.2. Let $X$ and $Y$ be any two spaces. If there is a homeomorphism from $\operatorname{cl}_{\beta X}(\beta X-X)$ onto $\operatorname{cl}_{\beta Y}(\beta Y-Y)$ which carries $R(X)$ onto $R(Y)$, then $K(X)$ is isomorphic to $K(Y)$.

Proof. Let $h$ be the given homeomorphism and $\Gamma: K(X) \rightarrow K(Y)$ the correspondence constructed in 1.4. By the symmetry of 1.4, $\Gamma$ is a bijection. That $\Gamma$ preserves order in both directions follows from the fact that $h[\mathscr{F}(\alpha X)]=\mathscr{F}[\Gamma(\alpha X)]$ and 2.1.

COROLlary 2.3. Let $X$ and $Y$ be two spaces with $|R(X)|=$ $|R(Y)| \leqq 1$. If $\beta X-X$ is homeomorphic to $\beta Y-Y$, then $K(X)$ is isomorphic to $K(Y)$.

Proof. In view of Magill's theorem, it suffices to consider $|R(X)|=$ $|R(Y)|=1$. Let $R(X)=\{p\}$ and $R(Y)=\{q\}$. Since $\operatorname{cl}_{\beta X}(\beta X-X)$ is the one point compactification of $\beta X-X$, open neighborhoods of $p$ in $\mathrm{cl}_{\beta X}(\beta X-X)$ are the complements of compact sets in $\beta X-X$. If $h$ is the given homomorphism, then $h$ carries compact sets onto compact sets. So it carries neighborhoods of $p$ onto neighborhoods of $q$ and vice versa. Hence if we let $k: \operatorname{cl}_{\beta X}(\beta X-X) \rightarrow \operatorname{cl}_{\beta Y}(\beta Y-Y)$ extend $h$ by $k(p)=q$, then $k$ is a homeomorphism and $k[R(X)]=R(Y)$. The result now follows from 2.2.

The next result follows from a well known exercise [3: 9K]. 
LEMmA 2.4. For any space $Y$ and any compactification $\alpha Y$, there is a pseudo-compact space $X$ such that $Y$ is homeomorphic to $\beta X-X$ and $\alpha Y-Y$ is homeomorphic to $R(X)$.

THEOREM 2.5. For each space $Y$, there is a pseudocompact space $X$ such that $K(Y)$ is isomorphic to $K(X)$.

Proof. As in the construction for 2.4, let $W$ be the ordinals less than the first uncountable ordinal $\omega_{1}$ and $W^{*}$ be its compactification. Set $X=\left[W^{*} \times \operatorname{cl}_{\beta Y}(\beta Y-Y)\right]-\left[\left\{\omega_{1}\right\} \times(\beta Y-Y)\right]$. Then $X$ is pseudocompact, $R(X)=\left\{\omega_{1}\right\} \times R(Y)$ and $\beta X-X=\left\{\omega_{1}\right\} \times(\beta Y-Y)$. The result now follows from 2.2 .

3. k-absolute spaces. A space is called compactly generated, or a $k$-space, if every set whose intersection with every compact set is compact is itself closed. To each space $X$ we may associate a unique $k$-space $\mathscr{K} X$ with the same underlying set and the same compact sets by requiring that the closed sets be precisely those whose intersection with every compact set is compact. It follows that $X$ is a $k$-space if and only if $X=\mathscr{K} X$.

Definition 3.1. $X$ is a $k$-absolute space if $\beta X-X$ is a $k$-space. This terminology is motivated by

THEOREM 3.2. For any space $X$, the following are equivalent:

(a) $\beta X-X$ is a k-space.

(b) For every compactification $\alpha X, \alpha X-X$ is a k-space.

(c) There exists a compactification $\alpha X$ such that $\alpha X-X$ is a $k$-space.

Proof. Use the fact that the restriction to $\beta X-X$ of the Cech map of $\alpha X$ is perfect (i.e., closed, continuous, onto and the pre-image of each point is compact), and the fact that if $f: V \rightarrow W$ is a perfect map, then $V$ is a $k$-space if and only if $W$ is a $k$-space [1: Theorem 8]. $k$-absolute space include, but are not restricted to, locally compact spaces, realcompact spaces (N. Noble [6]) and spaces with compact $R(X)$. Some examples showing the independence of these classes are considered in $\S 4$.

THEOREM 3.3. Let $X$ and $Y$ be any two spaces. If $\Gamma: K(X) \rightarrow$ $K(Y)$ is an isomorphism, then there is a homeomorphism $f: \mathscr{K}(\beta X-$ $X) \rightarrow \mathscr{K}(\beta Y-Y)$ such that for each $\alpha X$ in $K(X), \mathscr{F}[\Gamma(\alpha X)] \cap$ $(\beta Y-Y)=\{f[H]: H \in \mathscr{F}(\alpha X) \cap(\beta X-X)\}$. There are two such homeomorphisms if $|\beta X-X|=|\beta Y-Y|=2$; otherwise the home- 
omorphism is unique.

Proof. $f: V \rightarrow W$ is a bijection which preserves compact sets in both directions if and only if $f: \mathscr{K} V \rightarrow \mathscr{K} W$ is a homeomorphism. The proof now, with only minor changes, is that of K. D. Magill [5: Theorem 1].

CoRollary 3.4. Let $X$ and $Y$ be any two k-absolute spaces. If $K(X)$ is isomorphic to $K(Y)$, then $\beta X-X$ is homeomorphic to $\beta Y-Y$.

An example showing the converse of this corollary to be false is found in the following section. An example has been obtained by T. Thrivikraman [7] of a pair of spaces, one of which is $k$-absolute and the other is not, with $K(X)$ isomorphic to $K(Y)$, yet $\beta X-X$ not homeomorphic to $\beta Y-Y$.

\section{Examples.}

(A) k-absolute spaces.

(a) The rational numbers $Q$ form a realcompact, thus $k$-absolute space which is nowhere locally compact. Hence $R(X)=Q$ is not compact.

(b) Let $X$ be the ordinals $\leqq \omega_{1}$ with the discrete topology except at $\omega_{1}$, which has a neighborhood base of tails. Then $X$ is realcompact and $R(X)=\left\{\omega_{1}\right\}$ is compact.

(c) If $W$ is the set of ordinals $<\omega_{1}$ with the interval topology and $N$ is the positive integers, then $W \times N$ is locally compact, yet neither realcompact nor pseudocompact. (Not realcompact follows from the fact that closed subsets of realcompact spaces are realcompact, and $W \times N$ contains closed copies of $W$ ).

(d) To construct a class of $k$-absolute spaces which are neither locally compact nor realcompact, let $Y$ be any $k$-space and as in 2.4, let $X=W^{*} \times \beta Y-\left\{\omega_{1}\right\} \times Y$. This is a $k$-absolute, pseudocompact and not compact, hence not realcompact space. $R(X)$ is homeomorphic to $\beta Y-Y$, hence it is compact if and only if $Y$ is locally compact. NoTE: $X$ is locally compact if and only if $Y$ is compact.

(B) A pair of $k$-absolute spaces $X$ and $Y$ with $\beta X-X$ homeomorphic to $\beta Y-Y$, yet $K(X)$ and $K(Y)$ not isomorphic. Let $T=$ $(0,1)$ under its usual topology, $T^{*}$ its one point compactification and $T^{* *}$ its two point compactification. Write $T^{*}-T=\{a\}$ and $T^{* *}-$ $T=\{b, c\}$.

Set $X=W^{*} \times T^{*}-\left\{\omega_{1}\right\} \times T$, so $R(X)=\left\{\left(\omega_{1}, a\right)\right\}$.

Set $Y=W^{*} \times T^{* *}-\left\{\omega_{1}\right\} \times T$, so $R(Y)=\left\{\left(\omega_{1}, b\right),\left(\omega_{1}, c\right)\right\}$. So $|R(X)| \neq|R(Y)|$, yet $\beta X-X=\beta Y-Y=\left\{\omega_{1}\right\} \times T$, which is a $k$ space. 
Place the following compact partition on $\beta X-X$ : for each $r, 0<$ $r<1 / 2$, let $F_{r}=\left\{\left(\omega_{1}, r\right),\left(\omega_{1}, 1-r\right)\right\} ;$ choose $t_{r} \notin \beta X$ and set $\alpha X=$ $\left[\beta-\bigcup_{r} F_{r}\right] \cup\left\{t_{r}: 0<r<1 / 2\right\}$. Define the map $f: \beta X \rightarrow \alpha X$ by $f(x)=x$ if $x \in X$ and $f(x)=t_{r}$ if $x \in F_{r}$ and $f\left(\omega_{1}, 1 / 2\right)=\left(\omega_{1}, 1 / 2\right)$. If $G \leqq \beta X-X$, then $f^{\leftarrow} \circ f[G]=G$ if and only if $G$ is symmetric with respect to $\left(\omega_{1}, 1 / 2\right)$. Place the quotient topology with respect to $f$ on $\alpha X$. To show $\alpha X$ is Hausdorff, we apply (c) of Lemma 1.0.

Let $x \in X-\{a\}$ and $U$ be an open set of $\beta X$ such that $x \in U$. Then set $V=U \cap X-\{a\}$. So $f^{-} \circ f[V]=V$, which is an open set in $\beta X$, and $p \in V=f[V] \subseteq U$.

If $U$ is a $\beta X$-open neighborhood of $a$, then $U \cap \beta X-X \supseteqq\left\{\omega_{1}\right\} \times$ $(0,1)-\left\{\omega_{1}\right\} \times[d, e]$ for some $[d, e] \subseteq(0,1)$. Choose $\varepsilon>0$ so that $[d, e] \subseteq[\varepsilon, 1-\varepsilon] \subseteq(0,1)$.

Then $\left\{\omega_{1}\right\} \times(0,1)-\left\{\omega_{1}\right\} \times[\varepsilon, 1-\varepsilon]$ is open in $\beta X-X$, so there exists a $\beta X$-open set $H$ such that $H \cap \beta X-X$ equals this set. Let $V=$ $f[U \cap H]$. Since $U \cap H \cap \beta X-X$ is symmetric with respect to $\left(\omega_{1}, 1 / 2\right)$ we see that

$$
\begin{aligned}
f^{\leftarrow}[V] & =f^{\leftarrow} \circ f[(U \cap H \cap \beta X-X) \cup(U \cap H \cap X)] \\
& =(U \cap H \cap \beta X-X) \cup f^{\leftarrow} \circ f(U \cap H \cap X) \\
& =U \cap H \leqq U .
\end{aligned}
$$

Therefore $V$ is open in $\alpha X, a \in V$ and $f^{-}[V] \subseteq U$.

If $t_{r} \in \alpha X-X$, then $f^{\leftarrow}\left(t_{r}\right)=F_{r}$. Let $U$ be a $\beta X$-open neighborhood of $F_{r}$. Then $U \cap \beta X-X$ contains $\left(\omega_{1}, r\right)$, so there exists an $\varepsilon_{1}>0$ such that $\left\{\omega_{1}\right\} \times\left(r-\varepsilon_{1}, r+\varepsilon_{1}\right) \subseteq U \cap \beta X-X$. In the same way, there exists an $\varepsilon_{2}>0$ such that $\left\{\omega_{1}\right\} \times\left(1-r-\varepsilon_{2}, 1-r+\varepsilon_{2}\right) \cong$ $U \cap \beta X-X$. Let $\varepsilon=\min \left(\varepsilon_{1}, \varepsilon_{2}\right)$. Then $\left[\left\{\omega_{1}\right\} \times(r-\varepsilon, r+\varepsilon)\right] \cup$ $\left[\left\{\omega_{1}\right\} \times(1-r-\varepsilon, 1-r+\varepsilon)\right]$ is an open set in $\beta X-X$. So there exists a $\beta X$-open set $H$ such that $H \cap \beta X-X$ is equal to this set. Let $V=f[U \cap H]$. Note $U \cap H \cap \beta X-X=H \cap \beta X-X$ is symmetric with respect to $\left(\omega_{1}, 1 / 2\right), f^{\leftarrow} \circ f[U \cap H \cap \beta X-X]=U \cap H \cap$ $\beta X-X$. Hence $f^{\leftarrow} \circ f[U \cap H]=U \cap H$ and $V$ is open in $\alpha X$. Since $F_{r} \subseteq U \cap H$, we have $t_{r} \in V$ and $f[V] \subseteq U$. So $\alpha X$ is Hausdorff and thus in $K(X)$.

Suppose $\Gamma: K(X) \rightarrow K(Y)$ is any isomorphism; then by 3.4 there is a homeomorphism $h: \beta X-X \rightarrow \beta Y-Y$ such that $\mathscr{F}[\Gamma(\alpha X)] \cap$ $(\beta Y-Y)=\{h[H]: H \in \mathscr{F}(\alpha X) \cap(\beta Y-Y)\}$. Notice that any homeomorphism from $(0,1)$ to $(0,1)$ must be monotone: our argument is the same whether $h$ is monotone increasing or monotone decreasing. So without loss of generality, suppose $h$ monotone increasing.

Write $\Gamma(\alpha X)=\alpha Y$, where $\alpha X$ is the previously constructed compactification of $X$ and let $g$ be the restriction of the Čech map of $\alpha Y$ to $\beta Y-Y$. Since $f: \beta X-X \rightarrow \alpha X-X$ is perfect, it follows 
that if $k=g \circ h \circ f^{\leftarrow}$, then $k$ is a homeomorphism from $\alpha X-X$ onto $\alpha Y-Y$. Consider the sequence $t_{n}=\left(\omega_{1}, 1 / n\right), n \geqq 2$, in $\beta X-X$. The image of this sequence in $\alpha X-X$, which we may write as $p_{n}=$ $f\left(t_{n}\right), n \geqq 2$, has $\lim p_{n}=a$. So $\left(p_{n}\right), n \geqq 2$, is a converging sequence in $\operatorname{cl}_{\alpha Y}(\alpha Y-Y)$. But in $\operatorname{cl}_{\beta Y}(\beta Y-Y), \lim h\left(\omega_{1}, 1 / n\right)=b$ and $\lim \left(\omega_{1}, 1-\right.$ $1 / n)=c$. Therefore $k\left(p_{n}\right), n \geqq 2$, converges to both $b$ and $c$ in $\mathrm{cl}_{\alpha Y}(\alpha Y-Y)$. Since in a Hausdorff space, no sequence can converge to more than one point, $\Gamma(\alpha X)$ must not be Hausdorff. So $\Gamma$ must not be an isomorphism and thus $K(X)$ and $K(Y)$ are not isomorphic.

\section{REFERENCES}

1. A. Arhangel'skii, Bicompact sets and the topology of spaces, Dokl. Akad. Nauk SSSR 150 (1962), 9 = Soviet Math. Dokl., 4 (1963) 561-564.

2. N. Boboc and Gh. Siretchi, Sur la compactification d'un espace topologique, Bull. Math. Soc. Sci., Math. Phys. R. P. Roumaine (N. S.) 5 (53) (1961).

3. L. Gillman and M. Jerison, Rings of Continuous Functions, D. Van Nostrand Co., Inc., Princeton, N. J., 1960.

4. K. D. Magill, Jr., A note on compactifications, Math. Zeitschr., 94 (1966), 322-5.

5. - The lattice of compactifications of a locally compact space, Proc. London Math. Soc., 18 (1968), 231-44.

6. N. Noble, k-spaces and some generalizations, Doctoral Dissertation, University of Rochester, June 1967.

7. T. Thrivikraman, On the lattices of compactifications, J. London Math. Soc., 4 (1972), 711-717.

Received September 30, 1971 and in revised form September 27, 1972. The results of this paper are based on part of a doctoral dissertation submitted to the University of Kentucky in April, 1969. The author wishes to acknowledge the guidance and encouragement of his mentor, Dr. John E. Mack.

The University of Manitoba

WinNipeg, Manitoba, CaNada 


\section{PACIFIC JOURNAL OF MATHEMATICS}

\section{EDITORS}

\section{H. SAMELSON}

Stanford University

Stanford, California 94305

C. R. Новву

University of Washington Seattle, Washington 98105

\section{J. DuGundJI}

Department of Mathematics University of Southern California Los Angeles, California 90007

RICHARD ARENS

University of California Los Angeles, California 90024

\section{ASSOCIATE EDITORS}
E. F. BECKENBACH
B. H. NeumanN
F. WOLF
K. YoSHIDA

\section{SUPPORTING INSTITUTIONS}

\author{
UNIVERSITY OF BRITISH COLUMBIA \\ CALIFORNIA INSTITUTE OF TECHNOLOGY \\ UNIVERSITY OF CALIFORNIA \\ MONTANA STATE UNIVERSITY \\ UNIVERSITY OF NEVADA \\ NEW MEXICO STATE UNIVERSITY \\ OREGON STATE UNIVERSITY \\ UNIVERSITY OF OREGON \\ OSAKA UNIVERSITY
}

\author{
UNIVERSITY OF SOUTHERN CALIFORNIA \\ STANFORD UNIVERSITY \\ UNIVERSITY OF TOKYO \\ UNIVERSITY OF UTAH \\ WASHINGTON STATE UNIVERSITY \\ UNIVERSITY OF WASHINGTON \\ $*{ }^{*}$
AMERICAN MATHEMATICAL SOCIETY
NAVAL WEAPONS CENTER
}

The Supporting Institutions listed above contribute to the cost of publication of this Journal, but they are not owners or publishers and have no responsibility for its content or policies.

Mathematical papers intended for publication in the Pacific Journal of Mathematics should be in typed form or offset-reproduced, (not dittoed), double spaced with large margins. Underline Greek letters in red, German in green, and script in blue. The first paragraph or two must be capable of being used separately as a synopsis of the entire paper. The editorial "we" must not be used in the synopsis, and items of the bibliography should not be cited there unless absolutely necessary, in which case they must be identified by author and Journal, rather than by item number. Manuscripts, in duplicate if possible, may be sent to any one of the four editors. Please classify according to the scheme of Math. Rev. Index to Vol, 39. All other communications to the editors should be addressed to the managing editor, Richard Arens, University of California, Los Angeles, California, 90024.

50 reprints are provided free for each article; additional copies may be obtained at cost in multiples of 50 .

The Pacific Journal of Mathematics is issued monthly as of January 1966. Regular subscription rate: $\$ 48.00$ a year (6 Vols., 12 issues). Special rate: $\$ 24.00$ a year to individual members of supporting institutions.

Subscriptions, orders for back numbers, and changes of address should be sent to Pacific Journal of Mathematics, 103 Highland Boulevard, Berkeley, California, 94708.

PUBLISHED BY PACIFIC JOURNAL OF MATHEMATICS, A NON-PROFIT CORPORATION

Printed at Kokusai Bunken Insatsusha (International Academic Printing Co., Ltd.), 270, 3-chome Totsuka-cho, Shinjuku-ku, Tokyo 160, Japan. 


\section{Pacific Journal of Mathematics}

\section{Vol. 44, No. $2 \quad$ June, 1973}

Tsuyoshi Andô, Closed range theorems for convex sets and linear liftings . . . . . . 393

Richard David Bourgin, Conically bounded sets in Banach spaces . . . . . . . . . 411

Robert Jay Buck, Hausdorff dimensions for compact sets in $R^{n} \ldots \ldots \ldots \ldots \ldots \ldots . \ldots 421$

Henry Cheng, A constructive Riemann mapping theorem ................ 435

David Fleming Dawson, Summability of subsequences and stretchings of

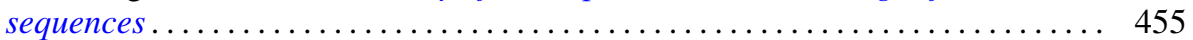

William Thomas Eaton, A two sided approximation theorem for 2-spheres ....... 461

Jay Paul Fillmore and John Herman Scheuneman, Fundamental groups of compact complete locally affine complex surfaces ....................... 487

Avner Friedman, Bounded entire solutions of elliptic equations . . . . . . . . . . . 497

Ronald Francis Gariepy, Multiplicity and the area of an $(n-1)$ continuous

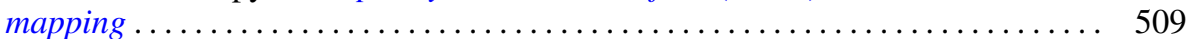

Andrew M. W. Glass, Archimedean extensions of directed interpolation groups . . . . 515

Morisuke Hasumi, Extreme points and unicity of extremum problems in $H^{1}$ on

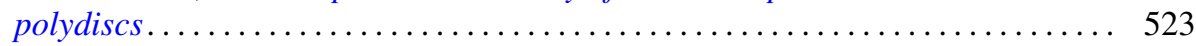

Trevor Ongley Hawkes, On the Fitting length of a soluble linear group . . . . . . 537

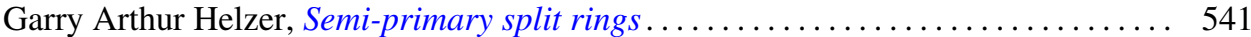

Melvin Hochster, Expanded radical ideals and semiregular ideals . . . . . . . . . 553

Keizō Kikuchi, Starlike and convex mappings in several complex variables . . . . . . 569

Charles Philip Lanski, On the relationship of a ring and the subring generated by its

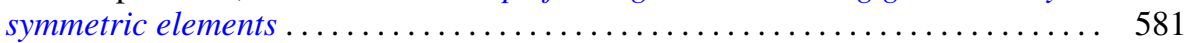

Jimmie Don Lawson, Intrinsic topologies in topological lattices and semilattices ........................................... 593

Roy Bruce Levow, Counterexamples to conjectures of Ryser and de Oliveira ...... 603

Arthur Larry Lieberman, Some representations of the automorphism group of an infinite continuous homogeneous measure algebra ..........

William George McArthur, $G_{\delta}$-diagonals and metrization theorems $\ldots .$.

James Murdoch McPherson, Wild arcs in three-space. II. An invariant of

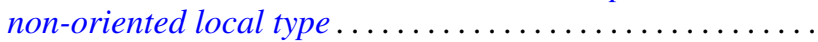

H. Millington and Maurice Sion, Inverse systems of group-valued measures ...

C. Edward Moore, Concrete semispaces and lexicographic separation of convex

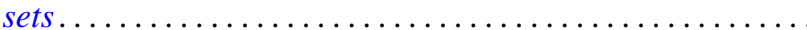

Jingyal Pak, Actions of torus $T^{n}$ on $(n+1)$-manifolds $M^{n+1}$.

Merrell Lee Patrick, Extensions of inequalities of the Laguerre and Turán type . . . . 675

Harold L. Peterson, Jr., Discontinuous characters and subgroups of finite index. . . . 683

S. P. Philipp, Abel summability of conjugate integrals . . . . . . . . . . . . . 693

R. B. Quintana and Charles R. B. Wright, On groups of exponent four satisfying an

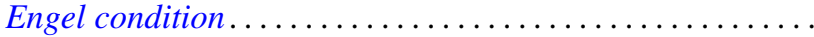

Marlon C. Rayburn, On Hausdorff compactifications. . . . . . . . . .

Martin G. Ribe, Necessary convexity conditions for the Hahn-Banach theorem in

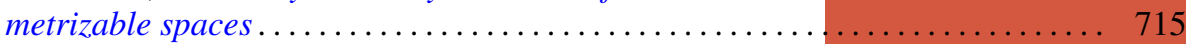

Ryōtarō Satō, On decomposition of transformations in infinite measure spaces .... 733

Peter Drummond Taylor, Subgradients of a convex function obtained from a

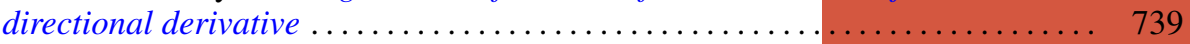

James William Thomas, A bifurcation theorem for $k$-set contractions . . . . . . . . 749 Clifford Edward Weil, A topological lemma and applications to real functions . . . . 757

Stephen Andrew Williams, A nonlinear elliptic boundary value problem . . ....... 767

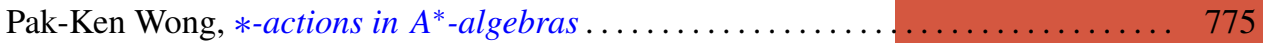

